\title{
Valoración económica del bosque nativo afectado por la introducción del castor americano en Tierra del Fuego
}

\author{
Economic valuation of native forest affected by the North American beaver \\ (Castor canadensis) in Tierra del Fuego \\ Aracely Soto Simeone a, Sergio Soza-Amigo a* \\ *Autor de correspondencia: ${ }^{a}$ Universidad de Magallanes, Facultad de Ciencias Económicas y Jurídicas, \\ Casilla 113-d, Punta Arenas, Chile, sergio.soza@umag.cl
}

\begin{abstract}
SUMMARY
How much is the economic value of native forest affected by the North American beaver in Tierra del Fuego? That was the question which led to determine the value given by society to forest resources affected by the introduction of the North American (or Canadian) beaver in conservation areas in Tierra del Fuego Province. The contingent valuation technique was applied and 396 persons of the economically active population from Punta Arenas and Porvenir were examined. This research found that $18.7 \%$ of the value placed on these resources is an option value, $48.8 \%$ corresponds to a heritage value, $17.1 \%$ falls on the existence value and $15.5 \%$ is attributed to their direct and indirect use values; thus, bringing its total valuation to \$4,864,507.534 (Chilean pesos, 2011) per year.
\end{abstract}

Key words: contingent valuation, compensating variation criterion, direct use value, indirect use value, non-use value.

\section{RESUMEN}

¿Cuál es el valor económico del bosque nativo afectado por la introducción del castor americano en Tierra del Fuego? Con tal planteamiento se determinó el valor otorgado por la sociedad, a los recursos forestales afectados por la introducción del castor americano (Castor canadensis), localizados en la provincia de Tierra del Fuego (Chile). Se utilizó como método la técnica de valoración contingente, entrevistando a 396 personas económicamente activas de las ciudades de Porvenir y Punta Arenas. De la investigación realizada, se constató que el 18,66 \% del valor otorgado a estos recursos constituye un valor de opción, el 48,76 \% corresponde a un valor de herencia, el $17,11 \%$ recae sobre el valor de existencia y el 15,48 \%, se atribuye al valor de su uso directo e indirecto, ascendiendo su valoración total a US\$ 7.282.197 (\$ 4.864.507.534; pesos de 2011) por año.

Palabras clave: valoración contingente, criterio de variación compensada, valor de uso directo, valor de uso indirecto, valor de no uso.

\section{INTRODUCCIÓN}

Los ecosistemas forestales del archipiélago fueguino constituyen los ambientes boscosos más australes y prístinos del planeta, caracterizándose por su fragilidad y lenta recuperación ante cualquier perturbación dada la rigurosidad climática, insularidad y biogeografía en dicha latitud (Fesq-Martin et al. 2004).

En 1946, y con el propósito de ser utilizados como materia prima en la industria peletera argentina, 25 parejas de castor americano (Castor canadensis Kuhl) provenientes de Canadá fueron introducidas y posteriormente liberadas en la isla de Tierra del Fuego (río Claro, sector noreste del lago Fagnano). Desde entonces, la especie se ha dispersado a otras islas más pequeñas y al área continental, estimándose su población en territorio chileno en aproximadamente 61.300 individuos al año 2006 (SAG-Magallanes 2007).

Un componente en las transformaciones que sufre el medio ambiente, lo constituyen las especies invasoras, fenómeno que probablemente se verá agravado con el cam- bio climático y la globalización de los mercados (Mooney y Cleland 2001). Junto a ello, la internacionalización y crecimiento del volumen comercial y el turismo, en conjunto a la apertura comercial, generan un panorama aún más favorable para que estas se dispersen, originando variados costos. En tal contexto, surge la pregunta ¿Cuál es el valor económico del bosque nativo afectado por la introducción del castor americano en Tierra del Fuego?

Las alteraciones más evidentes provocadas por este roedor derivan del uso de recursos para su alimentación y construcción de madrigueras, documentándose según Lizarralde et al. (1996), como una dieta exclusivamente vegetariana al forrajear sobre las tres especies más características de la zona: guindo o coigüe de Magallanes (Nothofagus betuloides Blume), lenga (Nothofagus pumilio Krasser) y ñirre (Nothofagus antarctica Oersted). Briones et al. (2001) estimaron en 23.593 hectáreas el área teóricamente afectada en Tierra del Fuego. Del total de esta superficie un $57 \%$ (aproximadamente $13.448 \mathrm{ha}$ ) corresponde a bosque nativo, recayendo el $43 \%$ restante (10.145 ha) sobre 
matorrales, praderas, humedales y otros. Considerando estos antecedentes, Soto (2011) estimó la cuantificación económica del daño biofísico realizado en \$ 47.045.609.169, dado por la pérdida por disminución de materias primas (\$43.060.939.069) y los costos de reforestación del área afectada (\$3.984.670.100).

El principal impacto del castor americano en el territorio austral es la remoción de bosque nativo a lo largo de zonas ribereñas constituyentes de gran parte de la hidrografía de Tierra del Fuego, las que se extienden dentro de 30 metros pero hasta 95 metros desde el curso de agua (Anderson et al. 2005). Estos recursos además corresponden a áreas preservadas para la biodiversidad y están calificados como bosques nativos de protección o conservación de acuerdo a lo establecido en el artículo 13 del Decreto Ley Nº 701 de 1974.

En ausencia de depredadores naturales, este roedor ha encontrado un ambiente propicio para su establecimiento y colonización, alterando; entre otros, el desarrollo normal de la actividad ganadera ovina propia de la zona. Así, su introducción se traduce en una amenaza creciente a las comunidades biológicas y ecosistemas australes, ocasionando asimismo perjuicios en el bienestar de la población derivados de la merma de recursos naturales generada.

Los aspectos económicos y sociales relacionados con las especies invasoras han recibido muy poca atención, probablemente por las dificultades de medir los detrimentos y beneficios generados por ellas en la sociedad. La mayor parte de los recursos afectados carece de precios de mercado y sus usos no sólo se limitan a uno actual y directo, además se deben incorporar los inherentes a usos potenciales.

El sistema de mercado, ofrece una solución al problema de la asignación de recursos al confluir en un mercado idealmente competitivo agentes económicos, que buscan maximizar sus funciones de utilidad generando precios mediante su interacción, en tanto que los consumidores, revelan sus preferencias a través de su disposición a pagar por bienes y servicios. Sin embargo, imperfecciones en el mercado tales como, la existencia de un conjunto de bienes y servicios que no poseen un mercado donde intercambiarse y por tanto, carecen de precios, desvirtúan esta situación ideal. No obstante, ello no implica que no tengan valor alguno: según el tipo de valor atribuido, se debe estimar un precio para ellos y así incluirlos en el sistema económico.

Pearce (1976) plantea la existencia de múltiples beneficios sociales asociados a los bosques en pie, siendo su valor económico total determinado por la suma de tres componentes principales: su valor de uso actual (beneficios directos e indirectos), el valor de opción (derivado de la seguridad en su provisión futura) y, el valor de existencia (asociado a principios éticos y ciertas formas de altruismo).

El propósito de valorar económicamente la contribución de ésta área protegida en Tierra del Fuego, radica en identificar, entender y dimensionar el bienestar que gran parte de la población de Magallanes obtiene de su existencia y mantenimiento. Cuantificar el bienestar aportado significa contar con una medida de los beneficios repor- tados, lo que permite analizar la conveniencia de distintas políticas para su aprovechamiento sustentable a fin de maximizar el bienestar de la población.

Se establecieron dos zonas como las potencialmente afectadas, las comunas de Porvenir y de Punta Arenas. En el caso de la primera, se evidencia un contacto directo de la población con el problema debido a la presencia del roedor en lugares aledaños, viéndose afectadas actividades tanto productivas (ganadería ovina) como de esparcimiento y desarrollo cognitivo-espiritual. Por su parte, para los habitantes de la comuna de Punta Arenas, existe la potencial amenaza de una invasión que condicionaría el abastecimiento de agua potable proveniente de Laguna Parrillar, la recreación y desarrollo de actividades al aire libre comúnmente realizadas en río San Juan y además, al igual que en Tierra del Fuego, la degradación de bosque nativo en áreas protegidas o de conservación con las consiguientes consecuencias que derivan de ello.

El concepto de disposición a pagar determinado por la variación compensada, resulta aplicable al problema que surge al no contar con precios de mercado para valorar los recursos forestales en áreas protegidas, cuyos flujos de beneficios obtenidos por la sociedad se han visto alterados a consecuencia de la degradación generada por la introducción de una especie invasora. La cifra monetaria asociada al valor económico de un activo ambiental no pretende representar un precio, sino un indicador monetario del valor que tiene para un individuo o para la sociedad el activo en cuestión. El método de valoración contingente (Ciriacy-Wantrup 1952) permite estimar el valor económico de recursos no transables, correspondiendo a una técnica de no-mercado basada en el criterio de variación compensada propuesto por Hicks (1943), cuyo objetivo era cuantificar la pérdida de bienestar.

La variación compensada se define entonces como la cantidad máxima que los individuos están dispuestos a pagar por un cambio favorable que les permita mantener su nivel de bienestar, el que podría empeorar de no realizarse dichas mejoras dado que dispondrían de una menor cantidad de recursos a consumir (Axioma 3 de la teoría del consumidor: "más es preferible a menos").

Basados en lo expuesto, el objetivo de este trabajo es cuantificar el valor económico asignado por los habitantes de las comunas de Punta Arenas y Porvenir al bosque nativo afectado por la acción invasora del castor americano, planteándose como hipótesis que la introducción del castor americano origina pérdidas sociales asociadas a los diversos valores de uso y de no uso del recurso forestal. Para cumplir con lo propuesto, se hace uso del método de valoración contingente.

\section{MÉTODOS}

Durante los meses de agosto y septiembre de 2011 se aplicó una encuesta, en la que se preguntó acerca de la cantidad máxima de dinero que estarían dispuestos a pagar en forma mensual, para que sea destinada a la realización de medidas enfocadas a revertir el daño originado por el castor americano. Para estos efectos, la población de referencia 
estuvo compuesta de individuos económicamente activos y mayores de 15 años residentes en las ciudades de Punta Arenas y Porvenir, localidades más pobladas y próximas a las zonas afectadas por la presencia del castor americano. Esta elección se atribuye al hecho de que las personas cercanas al área de estudio tienden a ser más conscientes sobre la importancia de un servicio local, no sólo porque tienen una mayor facilidad de acceso, sino porque tienen una mayor información como consecuencia de su experiencia (Johnson et al. 2001), de modo tal que sus valores, actitudes y conocimiento son dependientes del daño causado (Ajzen 1991).

Punta Arenas es la capital de la región de Magallanes y la Antártica Chilena, presentaba una población de 119.496 habitantes, según el censo del año 2002, de la cual un 97 \% era urbano y un 3 \% rural. Su población económicamente activa y mayor de 15 años era de 41.683 personas. Por su parte, Porvenir contaba con 5.465 habitantes de los cuales 4.734 vivían en la zona urbana, y su población económicamente activa (mayor de 15 años) estaba compuesta por 2.839 personas.

Para conocer el valor que la sociedad otorgaba a los recursos afectados, la población objeto de estudio debía poseer ingresos (renta) y pertenecer a la población económicamente activa, debido a que son ellos quienes podrían pagar como contribuyentes, por la realización de un cambio destinado a mejorar la situación que afecta su bienestar a largo plazo, dada la cercanía geográfica con las zonas afectadas. A su vez, y en consonancia con la teoría económica, esta disposición a pagar estaría condicionada por la restricción presupuestaria de cada individuo. Dicha población, compuesta por 44.522 personas, estuvo representada por una muestra ${ }^{1}$ de 396 individuos pertenecientes a diversos estratos sociales (composición proporcional a las respectivas poblaciones económicamente activas determinadas inicialmente por comuna).

De acuerdo a los antecedentes recopilados en las encuestas, se pudo determinar que el rango de edad de dicha muestra varió entre los 22 y los 75 años. La edad promedio de los entrevistados fue de 39 años, con una desviación estándar de 12,2 años. Además, se indagó el ingreso socioeconómico total individual mensual, basado en la clasificación de grupos socio-económicos utilizada por la Asociación Chilena de Empresas de Investigación de Mercado; esto es, según los estratos que se muestran: ABC1, C2, C3, D y E (donde: ABC1: segmento alto; C2: medio alto; C3: medio; D: medio bajo; E: bajo). Gran parte de los encuestados se ubicaba en estratos socioeconómicos medios a altos, encontrándose la mayor cantidad de personas entrevistadas en el estrato C2, seguido por el estrato ABC1, C3, D y observándose un porcentaje cercano al 1 \% de personas de la muestra pertenecientes al estrato socioeconómico más bajo (E). En promedio, los entrevistados poseían un

Para la muestra se utilizó una varianza de 0,09 $\left(\mathrm{s}^{2}=0,09\right)$; error estándar de 0,015 (se $=0,015$ ) y una probabilidad de ocurrencia de 0,9 . ingreso familiar total mensual de US\$ 1.675 (\$ 1.119.139 en pesos del año 2011; ubicándolos en el estrato socioeconómico C2), representado por el 45,32 \% del total de la muestra, con una desviación estándar de \$ 486.321 y un mínimo de \$ 240.000 y un máximo que ascendía a la suma de $\$ 2.000 .000$. Por su parte, el 28,35 \% pertenecía al estrato ABC1, 16,20 \% al nivel socioeconómico C3, 9,11 \% correspondía al estrato $\mathrm{D}$, mientras que el 1,01 \% al estrato $\mathrm{E}$.

En la distribución de edades promedio por estrato, se encontró que en el grupo ABC1 era de 38 años, en el grupo C2 era de 40 años, en el estrato C3 era de 38 años, en el estrato D de 32 años y en el grupo E de 48 años.

En relación al nivel educacional, los encuestados fueron divididos en seis categorías: educación básica incompleta, educación básica completa, educación media incompleta, educación media completa, educación superior incompleta, educación superior completa (sea esta universitaria o técnica).

La metodología aplicada, buscó determinar la disposición a pagar total anual (individual y social), a partir del cálculo de la disposición a pagar muestral, obtenida de los montos expresados por los encuestados pertenecientes a los diversos niveles socioeconómicos anteriormente expuestos, que respondieron a la pregunta abierta ¿Cuánto dinero estaría dispuesto(a) a pagar mensualmente, a fin de llevar a cabo mejoras ambientales enfocadas a proteger y conservar la biodiversidad del bosque nativo afectado por el castor?

Para establecer lo anterior, se planteó en la encuesta el desglose de la disposición a pagar, a modo de presentar los diversos componentes del valor económico total determinado por los valores de uso directo e indirecto y de no uso, información que se generó a través de preguntas sobre situaciones específicas en que se veían reflejados los valores de opción, de legado o herencia y de existencia.

Para determinar la disposición a pagar muestral, se utilizaron las 395 respuestas que dieron un valor positivo a la pregunta de valoración en pesos, considerando lo propuesto por Freeman (1993) y Riera (1994), que plantean que en los modelos de valoración contingente, no se debieran consideran las respuestas que no otorgaron un valor a la situación hipotética planteada. Posteriormente, los valores entregados por las personas muestreadas se agregaron para calcular la disposición a pagar total mensual $\left(\mathrm{DAPT}_{\mathrm{m}}\right.$ ). Luego, se dividió este último valor por el total de respuestas válidas (RV), con lo que se obtuvo la disposición a pagar promedio por persona al mes $\left(\mathrm{DAPP}_{\mathrm{mi}}\right)$. Posteriormente se multiplicó la $\mathrm{DAPP}_{\mathrm{mi}}$ por 12, obteniéndose la disposición a pagar promedio anual individual (DAPT $\mathrm{ai}_{\mathrm{a}}$ ). Para calcular la disposición a pagar total mensual por parte de la sociedad $\left(\mathrm{DAPT}_{\mathrm{ms}}\right.$ ), se multiplicó la DAPP $\mathrm{mi}_{\mathrm{m}}$ por el total poblacional (44.522), para luego llevar dicha cifra a términos anualizados. Posteriormente, se desglosó esta contribución con el fin de obtener los valores de no uso (valor de opción, de herencia o legado y de existencia), así como el de uso actual directo e indirecto del bosque nativo. 


\section{RESULTADOS}

De acuerdo con la clasificación de nivel educacional, el mayor porcentaje se encontró en la educación superior completa con un $48 \%$, seguido por la educación superior incompleta con un $35 \%$, deduciéndose de lo anterior que un $83 \%$ de la muestra ha realizado algún tipo de estudios superiores independiente de si estos han sido terminados o no. En tercer lugar se encuentra el grupo de entrevistados que ha terminado su educación media (17 \%).

En primera instancia, se preguntó a los encuestados si a ellos les interesaba el medio ambiente, pregunta ante la cual un 100 \% respondió que sí. Posteriormente, se les pidió calificar la importancia que tiene el medio ambiente para ellos, presentando el mayor porcentaje de la muestra la clasificación de "valioso" con un 62,5 \%; seguida por "muy importante" (29,6 \%) e "importante" (7,9 \%), no encontrándose personas en las categorías "poco importante” y "no es importante".

En relación con la protección, conservación y mejoramiento del estado del bosque nativo afectado, se encontró que un alto porcentaje de los entrevistados (98,7 \%) le interesa mucho la protección y conservación de estos bosques, en contraste con un 1,3\% de la muestra al que le interesa poco. No se encontraron personas que se declaran indiferentes al problema.

Del total de encuestados, un 99,7 \% estaban dispuestos a pagar una cierta cantidad de dinero mensual. Esta contribución, en promedio, asciende a US\$13,63 (\$ 9.105) con una desviación estándar de US\$ 9,04 (\$ 6.039), encontrándose personas dispuestas a pagar desde US\$ 0,75 (\$ 500) hasta US\$ 44,91 (\$30.000) como máximo. El valor más propuesto fue US\$7,49 (\$5.000; 44,6 \%), seguido por US\$14,97 (\$10.000) con el 26,3\% de los valores planteados. Otros valores fueron US\$29,94 (\$20.000; 15,2 \%) y US\$ 44,91 (\$ 30.000; 4,6 \%).

La disposición a pagar total mensual (DAPT $\mathrm{m}_{\mathrm{m}}$ ), ascendió a US\$ 5.384 (\$ 3.596.500). Por su parte la disposición a pagar promedio por persona al mes (DAPP ${ }_{\text {mi }}$ ), se estimó en US\$13,63 (\$ 9.105). La disposición a pagar promedio anual individual (DAPT ai ), resultó ser de US\$ 163,56 (\$ 109.261). Finalmente, la disposición a pagar por el total de los encuestados se estableció en US\$ 64.608 (\$ 43.158.000), coincidiendo este último valor con el excedente del consumidor (EC), dado por la diferencia entre la máxima disposición a pagar y lo que realmente se paga. Como en este caso los individuos no aportaban a un fondo mensual para la conservación del bosque afectado, el excedente del consumidor quedó establecido por la disposición a pagar promedio anual.

La disposición a pagar en forma mensual por parte de la sociedad(DAPT ${ }_{\mathrm{ms}}$ ), ascendióa US\$606.850 (\$405.375.628). Así, la disposición a pagar total anual por la sociedad (DAPT $T_{\text {as }}$ ) se calculó en US\$ 7.282.197 (\$ 4.864.507.534).

Considerando las preferencias de los contribuyentes, un 18,7 \% (US\$ 1.358.534; \$ 907.500.905) de su disposición a pago, se asignó a la conservación de dichos bosques para disfrutarlos y visitarlos en el futuro (valor de opción); un 48,8 \% (US\$ 3.550.880; \$ 2.371.987.924), se destinó a la conservación para futuras generaciones (valor de herencia); un 17,1 \% (US\$ 1.245.660; \$ 832.101.039) se asignó como valor de existencia (es decir, se valoró positivamente la existencia del bosque) y finalmente, un 15,5\% (US\$ 1.127.122; \$ 752.917.666) correspondió a un valor de uso directo e indirecto, lo que se interpreta como un uso tanto para los individuos como sus semejantes, para fines recreativos, culturales, científicos, educativos, entre otros.

Desde la perspectiva socioeconómica, se observó que las personas pertenecientes al estrato $\mathrm{ABC} 1$, presentaron una disposición promedio mensual a pagar de US\$22,20 (\$14.828), y el estrato socioeconómico C2 de US\$10,36 (\$ 6.919) de acuerdo a la valoración realizada. No obstante, los niveles C3, D y E, declararon estar dispuestos a pagar montos de dinero proporcionalmente más altos en relación a sus ingresos, siendo estos de US\$ 10,01 (\$ 6.688) en el primero; en el segundo US\$10,31 (\$ 6.889) y US\$2,99 (\$ 2.000) en el tercero.

Respecto a las pérdidas de bienestar, quienes pertenecían a los estratos socioeconómicos de más altos ingresos (ABC1), estuvieron dispuestos a pagar por los recursos afectados montos superiores a los que declararon los demás segmentos, correspondiendo su aporte mensual al 1,59 \% de sus ingresos. No obstante lo anterior, las cantidades de dinero propuestas por quienes pertenecían al nivel $\mathrm{D}$, fueron proporcionalmente mayores en relación a los ingresos mensuales que perciben, siendo estas equivalentes a un 3,13 \% de sus ingresos. En tanto, que la relación aporte/ingresos mensuales de los segmentos C2, C3 y E correspondió a un $1,30 \%$, un $1,89 \%$ y un 1,25 \%, respectivamente.

\section{DISCUSIÓN}

Un aspecto importante a destacar, relacionado con la disposición a pagar de la población analizada respecto de sus ingresos, reside en la posibilidad de encontrar que aquellos individuos pertenecientes a los estratos sociales más acomodados no fueran precisamente quienes estarían dispuestos a pagar montos mayores. Sin embargo, esta situación no se presentó, ya que en la mayoría de los casos los encuestados pertenecientes a los segmentos de más altos ingresos, declararon una mayor predisposición ante el pago por los bienes y servicios ambientales en cuestión.

En relación a la alta valoración que se tiene respecto a la protección, conservación y mejoramiento del estado del bosque nativo afectado, se explicarían según Pearce y Turner (1990) por: primero, el deseo de preservar un determinado bien para su disfrute por parte de futuras generaciones; segundo, la benevolencia hacia semejantes que lleva a valorar bienes porque ellos lo hacen (altruismo localizado); tercero, la simpatía -en su sentido más smithianopara con la población afectada por el deterioro de bienes ambientales, aun cuando no se tenga una relación directa con ellos (altruismo global); y finalmente, por la creencia en el derecho a la existencia de otras formas de vida, in- 
cluyendo por tanto a animales y plantas, conectándose esta postura con variantes de la ética ecocéntrica en que la naturaleza no-humana tiene un valor intrínseco e inherente, $\mathrm{y}$ posee por tanto derechos morales y naturales.

Observaciones a este método radican en que el uso de valoración contingente implica asumir que el valor de no uso puede ser claramente identificado y medido y que representa una proporción importante dentro del valor total que los individuos manifiestan hacia un bien (Lazo et al. 1992, Cameron 1992, Cicchetti y Wilde1992, Larson1992, 1993, Bishop y Welsh 1993). Cummings y Harrison (1995), en tanto, plantean que solo es posible observar el valor total de un bien y no las intenciones de los individuos con respecto a este valor. Finalmente, otros autores como Diamond (1996), Kahneman y Knetsch (1992), Diamond y Hausman (1994), Loomis y De Lacy (1992) argumentan que la valoración contingente no capta con exactitud y confiabilidad la valuación que los individuos realizan con respecto a un bien. De este modo, la técnica en cuestión se plantea como un método controversial, dado que se basa en lo que las personas harían y no en lo que se observa que los individuos realizan, situación que se constituye así en su principal fortaleza y debilidad a la vez.

Otras críticas se han enfocado en argumentar que las personas están poco familiarizadas con asignar valor a bienes o servicios ambientales, lo que puede llevar a que el monto asignado no sea precisamente el más adecuado. De igual modo, los individuos podrían estar respondiendo a las indagaciones realizadas influenciados por la expresión de sentimientos más que de valor (Cerda 2003).

Respecto al trabajo realizado y su comparación con otras experiencias similares en Chile, no se encontraron. La aplicación para el castor resultó ser única, dificultando por tanto el establecer si el valor determinado es alto, medio o bajo respecto a otras valoraciones. Sin embargo, en general para el caso de Chile, estudios donde se aplica la valoración contingente y que se pueden considerar similares dado que se aplican a recursos naturales son, entre otros, los realizados por Ávila (2005), donde establece el valor que la sociedad asigna a la conservación de los bosques de ruil (Nothofagus alessandrii Espinosa). Utilizando como muestra la población chilena mayor de 18 años con un margen de error similar al aquí empleado, determinó que las preferencias de los encuestados son para valor de uso un 45,0 \%, para valor de opción un 46,6 \% y un 8,4 \% para valor de existencia. Como se puede apreciar estos valores difieren de los aquí obtenidos, ya que la población analizada en este estudio valora en forma mínima el valor que se le asigna al bosque por concepto de uso (15,5\%). El valor de opción en ambos trabajos se aprecia de forma similar (46,6 versus 48,8\%) y finalmente para el valor de existencia, se observa que los encuestados en el estudio duplican los valores presentados por Ávila (2005) (17,1 versus 8,4 \%), diferencias que podrían ser atribuidas a la población muestreada.

En un estudio realizado por Muñoz (2000) se valoró económicamente el Monumento Natural Cerro Ñielol, estimándose una disposición a pagar promedio de $\$ 480.000$ por persona al año. Las características socioeconómicas más significativas fueron la edad y los ingresos de los entrevistados, los que se comportan de forma inversamente proporcional a la disposición a pagar, es decir, a medida que se incrementan dichas variables disminuye el valor medio de esta. En comparación con este estudio, se observa más homogeneidad en las respuestas.

Otras indagaciones como las presentadas en el trabajo de Molina (2001) consistentes en la valoración económica del Parque Nacional Tolhuaca, dan cuenta de una disposición a pagar promedio por persona que asciende a $\$ 429.675$ al año. Las variables más significativas y explicativas en este estudio fueron el total de años de educación, el ingreso familiar y el estado civil de los encuestados. En contraste, Barrientos (2000) al valorar económicamente el Parque Nacional Huerquehue estimó una disposición a pagar promedio individual de $\$ 941.039$ por año, dado por encuestados cuyos rangos de ingresos no presentan mayor variación perteneciendo estos a un mismo grupo socioeconómico. Como se puede observar, los resultados obtenidos en comparación a estos últimos dos estudios representan un punto medio entre ambos.

De acuerdo a lo que fue posible apreciar y considerando una tasa de respuesta suficientemente alta, las preferencias reveladas son contingentes al escenario en particular sobre el cual se construyó el mercado hipotético; por tanto, tales resultados pueden ser generalizados a la población de la cual se tomó la muestra con un determinado margen de error. El método aplicado ha permitido la medición de beneficios que no requieren el consumo o servicio directo del bien (valores de opción, de herencia y de existencia), siendo especialmente útil para este caso en particular, dada la carencia de información para efectos de estimar en qué medida se demandan los bienes y servicios ambientales que son objeto de estudio.

\section{CONCLUSIONES}

Los resultados obtenidos permiten concluir que la metodología aplicada exterioriza elementos de base psicosocial involucrados en el proceso de valoración de los sistemas naturales y sus componentes, y es perfectamente extrapolable a circunstancias similares.

En relación a la importancia que la población encuestada le asignó al interés por el medio ambiente, la gran mayoría le otorga una alta valoración al mismo. Así mismo, a un alto porcentaje de los entrevistados le interesa mucho la protección y conservación de estos bosques.

En relación al universo encuestado, prácticamente la totalidad de las personas manifiesta su disposición al pago. Respecto de la pérdida de bienestar y sobre la base de la metodología de la variación compensada, quienes pertenecen a estratos socioeconómicos de más altos ingresos (ABC1) estarían dispuestos a pagar por los recursos afectados montos superiores a los que declaran los demás segmentos, sin embargo, estos pagarían menos que los estratos D y C3 desde la perspectiva de la proporción del aporte respecto al ingreso. 
La alta disposición a pagar entre los diferentes estratos socioeconómicos podría atribuirse a factores educacionales, considerando el alto nivel educacional encontrado en el universo de encuestados.

Dada la disposición a pago y el alto interés detectado por la protección, conservación y mejoramiento del estado del bosque nativo, una estrategia que se podría aplicar es la realización de programas que involucren visitas de la comunidad a los bosques afectados, lo que debiera incrementar la sensibilidad social por conservar a estos bosques en su estado más prístino.

\section{AGRADECIMIENTOS}

Se reconocen y agradecen los financiamientos entregados por parte del Ministerio de Economía, Fomento y Turismo, Proyecto NS 100046 (Ciencia Regional y Políticas Públicas), de la Iniciativa Científica Milenio.

\section{REFERENCIAS}

Ajzen I. 1991.The theory of planned behavior.Organizational Behavior and Human Decision Processes 50(2): 179-211.

Anderson C, M Cienek, A Gutiérrez, R Rozzi. 2005. Biota terrestre y dulceacuícola - Expedición enero 2005. In Informe Técnico: Proyecto, diagnóstico, diseño y gestión del Parque Regional Tierra del Fuego. Chile Sustentable. Santiago, Chile. Ministerio de Bienes Nacionales. p. 41-162.

Ávila A. 2005. Evaluación económico-ambiental para determinar el valor monetario que la sociedad le asigna a la conservación de los bosques de Ruil (Nothofagus alessandrii Espinosa), a través del método de valoración contingente. Tesis Licenciado en Ciencias Forestales. Temuco, Chile. Universidad Católica de Temuco. 111 p.

Bishop R, M Welsh. 1993. The role of question order and respondent experience in contingent valuation studies. Journal of Environmental Economics and Management 25(1): 45-55.

Briones M, R Schlatter, A Wolodarsky, C Venegas. 2001. Clasificación ambiental para hábitats de Castor canadensis (Kuhl 1820, Rodentia), de acuerdo a características de cuencas en un sector de Tierra del Fuego. Anales del Instituto de la Patagonia 29 (2): 75-93.

Cameron T. 1992. Combining contingent valuation and travel cost data for the valuation of nonmarket goods. Land Economics 68 (3): 302-317.

Cerda C. 2003. Valoración Económica del Patrimonio Natural de la Reserva Nacional Lago Peñuelas. Tesis Magíster en Gestión y Planificación Ambiental. Santiago, Chile. Universidad de Chile. 75 p.

Cicchetti C, L Wilde. 1992. Uniqueness, irreversibility, and the theory of non-use values. American Journal of Agricultural Economics 74: 1121-1125.

Ciriacy-Wantrup SV. 1952. Resource conservation: economics and policies. Berkeley, USA. University of California Press. 395 p.

Cummings R, G Harrison. 1995. The measurement and decomposition of nonuse values: a critical review. Environmental and Resource Economics 5: 225-247.

Diamond P. 1996. Testing the Internal Consistency of Contingent Valuation Surveys. Journal of Environmental Economics and Management 30: 337-347.

Diamond P, J Hausman. 1994. Contingent valuation: is some number better than no number? The Journal of Economic Perspectives 8: 45-64.

Fesq-Martin M, A Friedmann, M Peters, J Behrmann, R Kilian. 2004. Late-glacial and Holocene vegetation history of the Magellanic rain forest in southwestern Patagonia, Chile. Vegetation History \& Archaeobotany 13(4): 249-255.

Freeman A. 1993. The measurement of environmental and resource values: theory and methods - resources for the future. Washington DC, USA. Business \& Economics.491 p.

Hicks J. 1943.The four consumer's surpluses. Review of Economic Studies 11: 31-41.

Johnson F, R Dunford, W Desvouges, M Banzhaf. 2001. Role of knowledge in assessing nonuse values for natural resource damages. Growth and Change 32(1): 43-68.

Kahneman D, J Knetsch. 1992. Valuing public goods: the purchase of moral satisfaction. Journal of Environmental Economics and Management 22(2): 57-70.

Larson D. 1992. Can non-use value be measured from observable behavior. American Journal of Agricultural Economics 74(5): 1114-1120.

Larson D. 1993. On measuring existence value. Land Economics 69(4): 377-388.

Lazo J, W Schulze, G McClelland, J Doyle. 1992. Can contingent valuation measure non-use values? American Journal of Agricultural Economics (December): 1126-1132.

Lizarralde M, G De Ferrari, J Escobar, S Alvarez. 1996. Effects of beaver (Castor canadensis) on the nutrients dynamics of the Southern Beech forest of Tierra del Fuego. Ecología Austral 6(2):101-105.

Loomis J, T De Lacy. 1992. Some Empirical Evidence on Embedding Effects in Contingent Valuation of Forest Protection. Journal of Environmental Economics and Management 199: 45-55.

Mooney H, E Cleland. 2001. The evolutionary impact of invasive species. Proceedings of the National Academy of Sciences 98: 5446-5451.

Pearce D. 1976. Economía Ambiental. México DF, México. Fondo de Cultura Económica. 258 p.

Pearce D, R Turner. 1990. Economics of the Natural Resources and the Environment. Londres, Inglaterra. Editorial Harvester Wheatsheaf. 378p.

Riera P. 1994. Manual de Valoración Contingente. Madrid, España. Instituto de Estudios Fiscales. 137 p.

SAG-Magallanes (Servicio Agrícola y Ganadero, Magallanes y Antártica Chilena, CL). 2007. Resumen Ejecutivo Informe de Gestión Período 2004-2007. Programa "Control de Especies Invasoras en Magallanes”. Punta Arenas, Chile. SAG-FONDEMA. 13 p.

Soto A. 2011. Cuantificación Económica del Daño Ambiental causado por la Introducción del Castor en Tierra del Fuego. Trabajo de Titulación Ingeniería Comercial. Punta Arenas, Chile. Universidad de Magallanes. 190 p. 